\title{
Effective Approach to Extract CMOS Model Parameters Based On Published Wafer Lot Data
}

\author{
Ashraf A. Osman ${ }^{1}$ and Amin B. Abdel Nabi ${ }^{2}$ \\ ${ }^{1}$ Department of Electrical Engineering, California State University at Sacramento, Sacramento, CA, USA \\ ${ }^{2}$ Department of Telecommunications Eng., Al-Nileen University, Khartoum, Sudan
}

\begin{abstract}
The VLSI electronic circuit designs have steadily grown in their capacity and complexity through the years. MOSIS fabrication services provide test data that designers can used to simulate their circuit designs. The provided test results are extracted from various lot wafers and BSIM3 or BSIM4 model card parameters in addition to technology parameters are provided. It may be cumbersome to ensure design functionality over the wide range of model set of parameters. In this paper, it is proposed to utilize the average model parameters to validate circuit design functionality. It can be shown through device characterization and simple circuit simulations that the average model parameters can provide a good representation of the wide range of supplied model parameters. This is specially attractive for students of circuit design classes where classroom and graduate research work were computing resources are limited. Utilizing average model parameters alleviate the need to run simulations over the large set of models from the fabrication facility.
\end{abstract}

Keywords: VLSI; Design; Automation; CAD; EDA; Higher Education, Circu it; Microelectronics.

\section{INTRODUCTION}

The VLSI electronic circuit designs have steadily grown in their capacity and complexity through the years. The circuit simulation based on technology test data is a core capability to ensure quality and functionality of circuit design. Established circuit design companies are well equipped with commercial and proprietary CMOS models. However, for educational institutes specially in developing countries the needs are massive. Circuit design students will have access to only published or granted set of CMOS device parameter models. One example provider of circuit manufacturing and model parameters is the MOSIS Integrated Circu it Fabrication Service[1]. MOSIS portal online site provides access to SPICE model parameter sets extracted through testing shuttles. The ability to enable circuit simulation as part of circuit design capability is critical to the whole circuit design and automation flow as desicribed by Osman et. al. with focus on developing country higher education institutes [2].

For teaching purposes, it is normal that a semiconductor modeling or circuit design class would require students to perform simulations using various circuit simulator tools. More advanced classes or project work would require class to complete circuit design projects going over steps of designing the circuitry, validating functionality, and in certain cases submit design for fabrication and test circuit performance post design and fabrication. Such task list would constitute the normal set of requirements for graduate level of research work.

The focus of this work is to scheme an approach that would enhance simulation capability for circuit design educational projects by extracting fewer set of model parameters to be used for design validity check. The approach would list technical steps to be followed to reach the minimu m required parameter set that would enable circuit testing over the valid technology spectrum. It is also expected to enhance the simulation by providing a fewer set of model parameters needed for simulations by student and instructors. This approach is expected to lead to faster turn around time for school projects to complete and with more confidence on circuit simulations. Moreover, the approach would be attractive to educational firms where less number of simulations would be needed on these student projects, hence, better use of limited computational resources available to developing countries standard educational institutes.

The proposed approach will be described and detailed in the following sections. It will also be run over a set of MOSIS CMOS model parameters on various technologies. 


\section{DESCRIPTION OF MOSIS TEST RESULTS AND SPICE PARAMETERS}

An example of fabrication services is provided by MOSIS Integrated Circuit Fabrication Service[1] which has a protal site that lists various test results extracted from many fabrication processes supplied to enable test chip designers from education institutes with circuit simulations.
For this work, a list of test parameters were copied from MOSIS portal for processes $0.5 \mathrm{um}, 0.35 \mathrm{um}$, $0.25 \mathrm{um}, 0.18 \mathrm{um}$, and $0.13 \mathrm{um}$. Model card were provided on level 49 BSIM 3 which is level 7 when using PSPICE circuit simulator. Test results reported in this paper are from the $0.13 \mathrm{um}$ technology with a total of 140 NMOS device model cards, and 140 PMOS model cards.

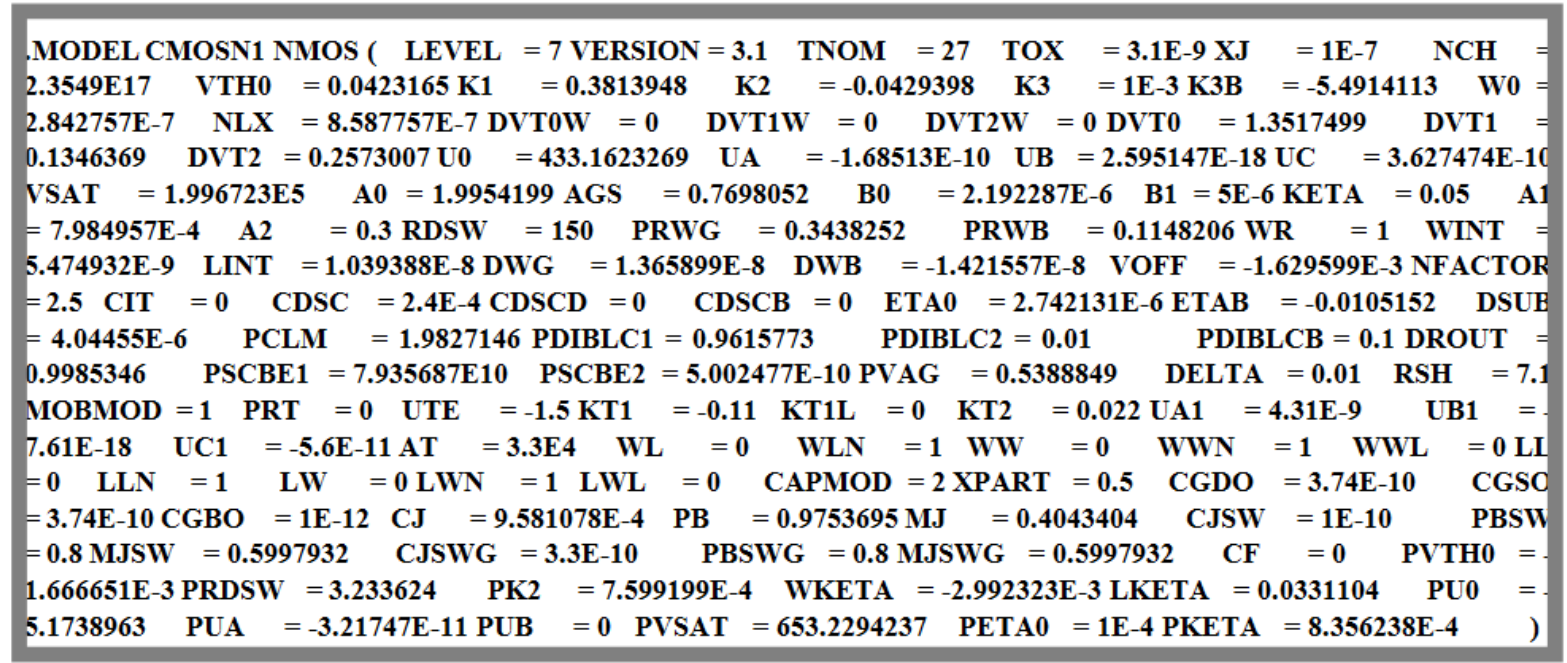

Figure 1. Sample of model cards for NMOS devices with model names CMOSN1 from the portal where 140 model cards NMOS and PMOS 0.13u m Technology were extracted.

\section{SIMULATION MODEL PARAMETERS PREPARATION STEPS}

To validate circuit design functionality, one would need to run simulations using all of the reported models and ensure functionality is falling within required specifications. For example, if one uses the $0.13 \mathrm{um}$ from MOSIS portal, a 140 NMOS and PMOS model cards would be used to run circuit simulations and ensure functioanlity for all of the model cards. This pose a real challange to simulate circuit functionality and would require an abundance of resources to accomplish.

It is imperative to scheme a method to reduce required simulation runs without sacrificing circuit functionality. The following steps are proposed to tackle such challenge and enable designers to test functionality over the wide range of models provided by test data:

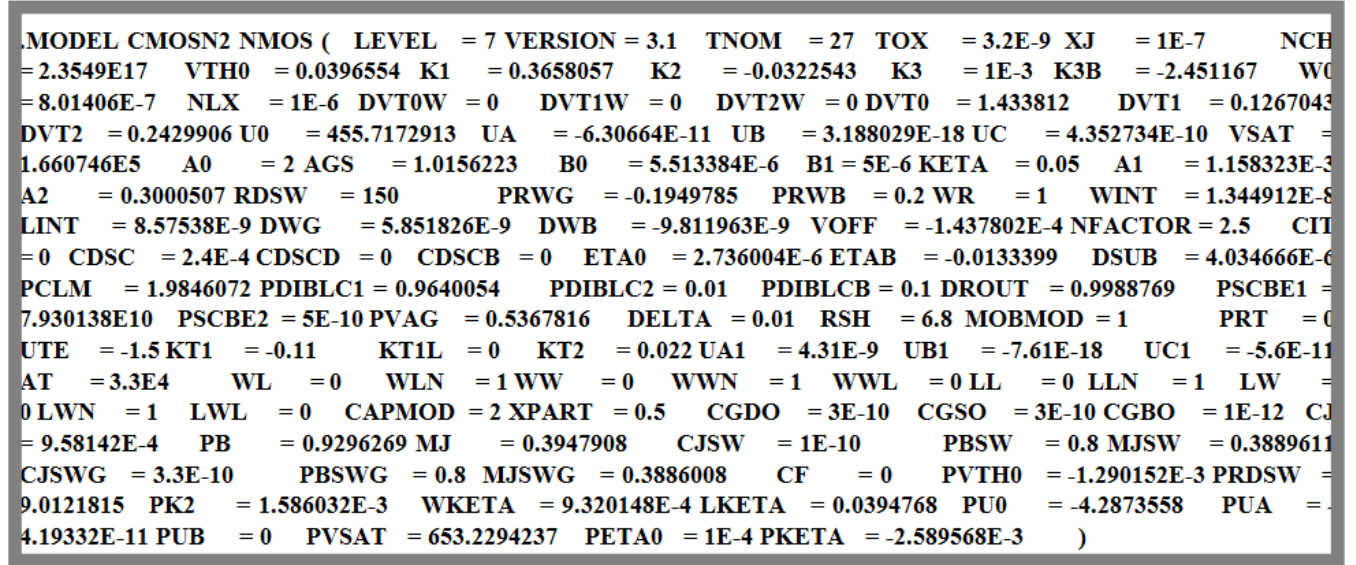

Figure 2. Sample of model cards for NMOS devices with model names CMOSN2 from the portal where 140 model cards NMOS and PMOS $0.13 \mathrm{um}$ Technology were extracted 


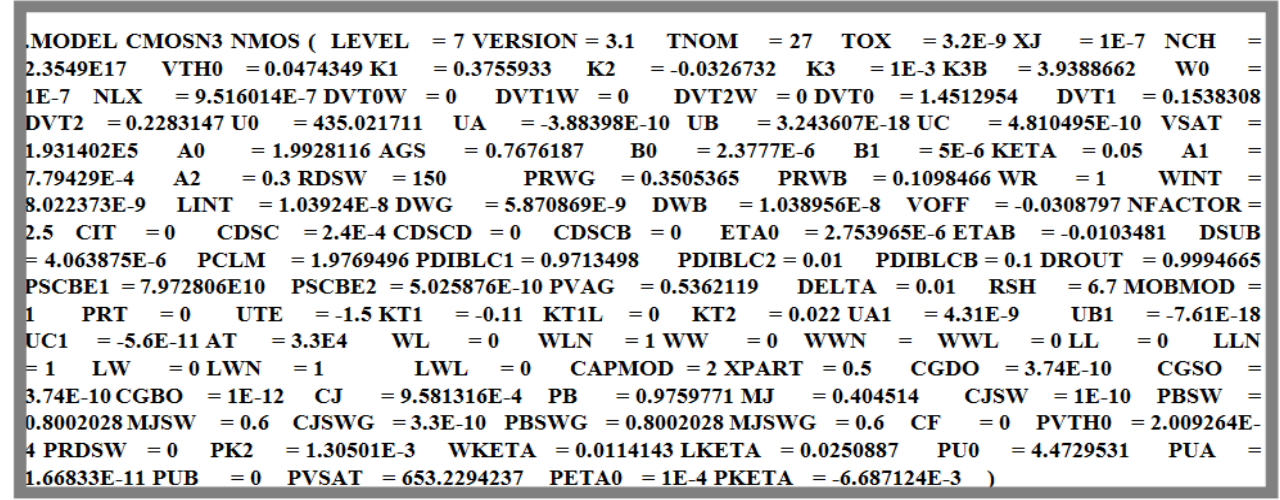

Figure 3. Sample of model cards for NMOS devices with model names CMOSN3 from the portal where 140 model cards NMOS and PMOS 0.13u m Technology were extracted.

1. Download model parameters from MOSIS portal and save test results in a text file

2. Develop a utility to:

a) Extract the BSIM model card for NMOS and PMOS devices from test data file.

b) Process model parameters and save in CSV, Excell format, or any tabular format for later processing.

3. Compute BSIM model card using the mathematical function of minimum (MIN), maximu m (MAX), and average (AVERAGE) value of all BSIM model parameters to produce new model cards.

4. Save the minimum, maximum, and average model parameter sets in their own model card with model name updated to reflect type of parameter set.

5. The average, min, and max model card can be used to run simulations to test circuit functionality.

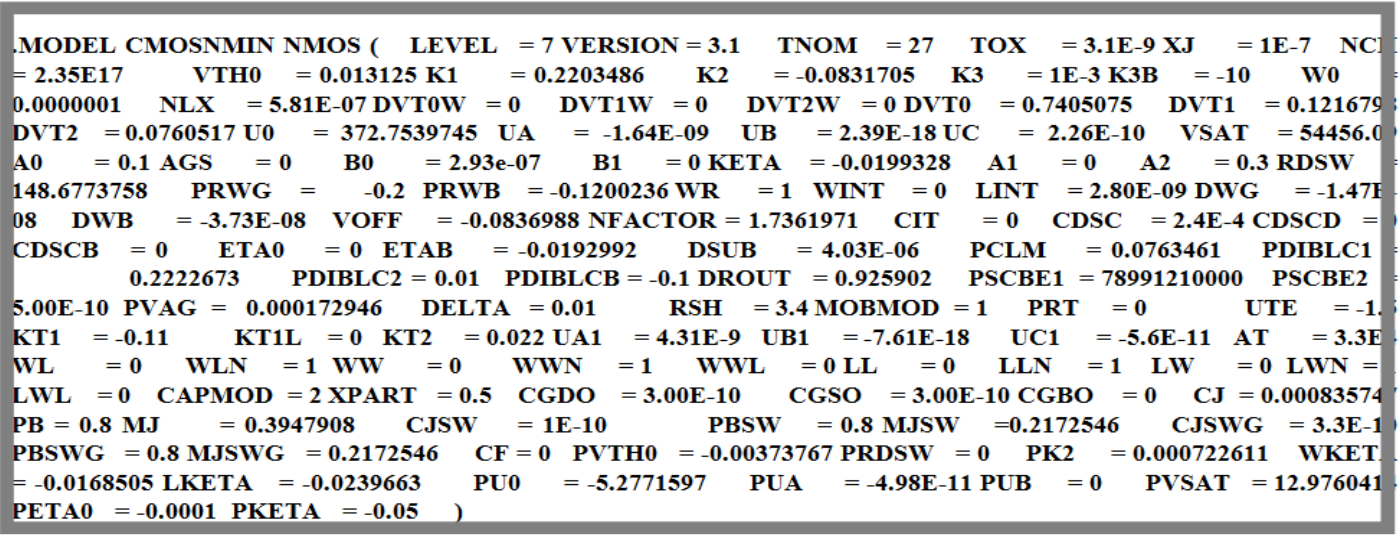

Figure 4. The min mu marameter model cards for NMOS devices named CMOSNMIN from the portal where 140 model cards NMOS and PMOS 0.13u m Technology were extracted.

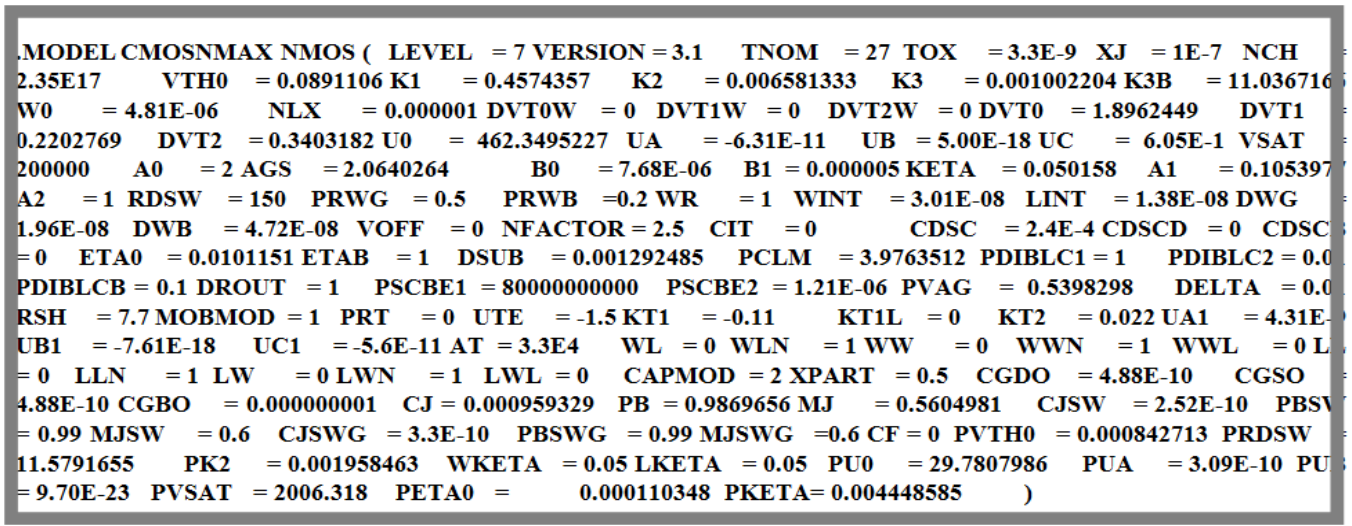

Figure 5. The maximu marameter model cards for NMOS devices named CMOSNMAX from the portal where 140 model cards NMOS and PMOS 0.13u m Technology were extracted. 
The proposed steps were executed on a the set of 140 model parameters from 0.13 um with NMOS and PMOS models as well as from other processes. The min, max, and average cards are computed used the listed steps above. The model cards CMOSN1, CMOSN2, CMOSN3 are 3 sample model cards from the complete set of avaliable model cards are shown in Fig1., Fig2, and Fig 3 respectively. The derived minimum parameter model card is derived and labelled with CMOSNMIN, and is shown in Fig. 4. The model card with maximum parameters model card and labelled with CMOSNMAX and is shown in Fig. 5.
The average model parameters are computed and listed in the model card with label CMOSA VG as shown in Fig. 6. These model card are used with PSPICE circuit simulator for testing and analys is and the model parameter LEVEL is set to 7 as required by the simulator.

\section{SIMULATION AND ANALYSIS}

To validate the extracted Min, Max, and Avgerge model cards accuracy and suitablity for circuit design and function testing, an experiment is devised with the following details:

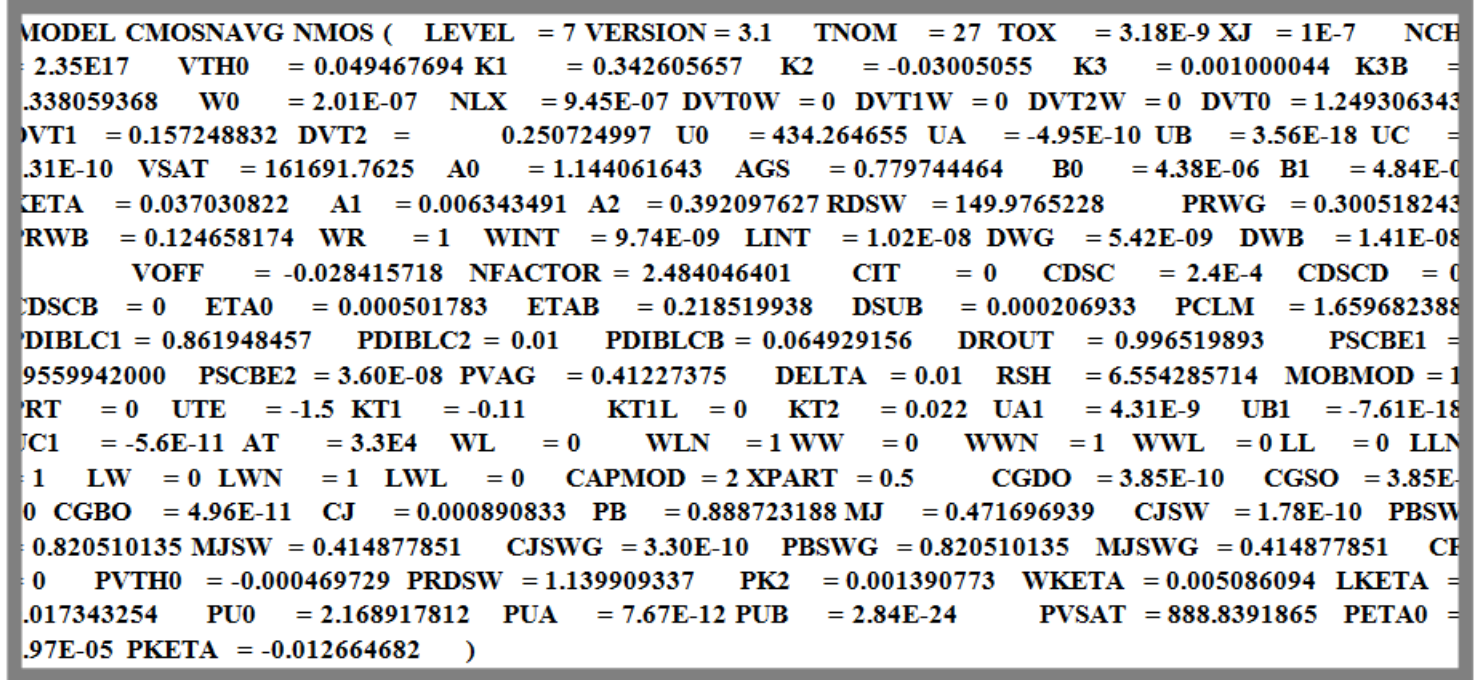

Figure 6. The average parameter model cards for NMOS devices named CMOSNA VG from the portal where 140 model cards NMOS and PMOS $0.13 \mathrm{um}$ Technology were extracted.

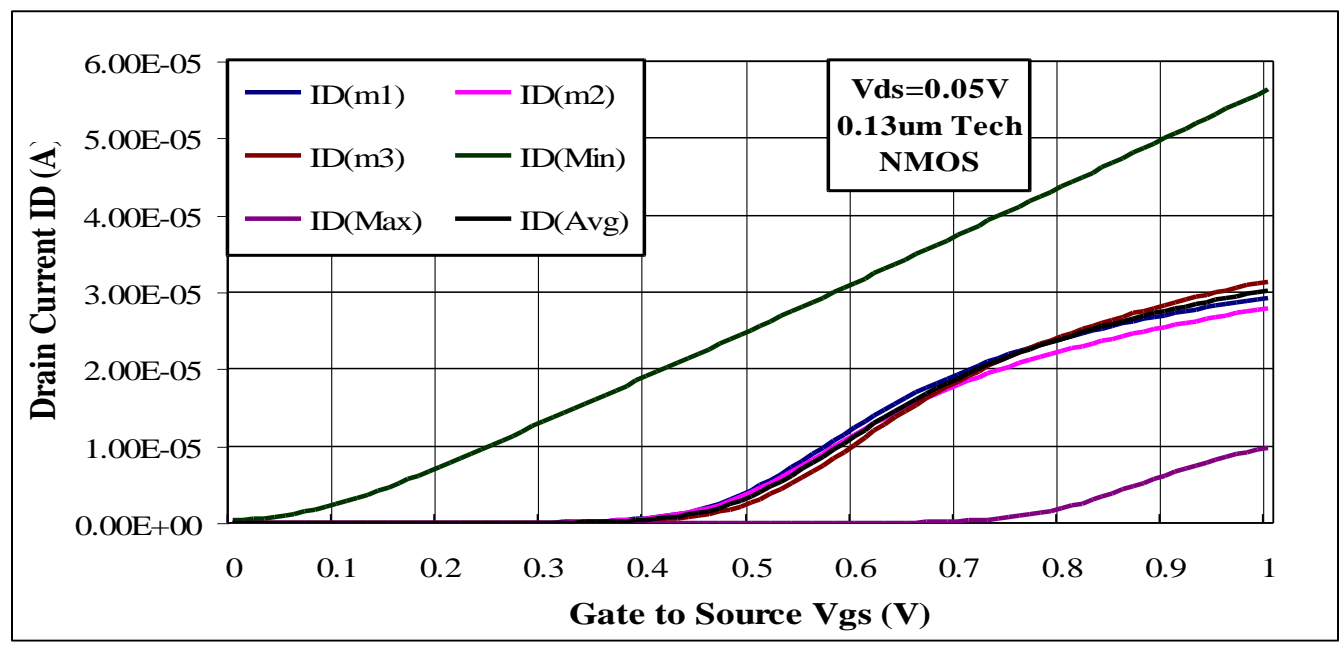

Figure 7: Linear region of NMOS device $(0.5 \mathrm{u} / 0.13 \mathrm{u})$ at low $\mathrm{Vds}=0.05 \mathrm{~V}$ and using model cards for CMOSN1, CMOSN2, CMOSN3 from the portal and the computed CMOSNMIN, CMOSNMAX, and CMOSNA VG model cards using the 140 model cards downloaded from MOSIS portal for NMOS and PMOS 0.13u m Technology. 
1. Test validity over a single device (NMOS, PMOS) characteristics. The extracted models were used to simulate NMOS and PMOS:

A) drain current Ids,

B) drain conductance Gds,

C) and gate trans conductance $\mathrm{Gm}$
D) Sweep of drain to source (Vds) bias

E) and sweep of gate to source (Vgs) bias

2. A simple inverter circu it is also used to check model validity by testing inverter DC transfer charactistics.

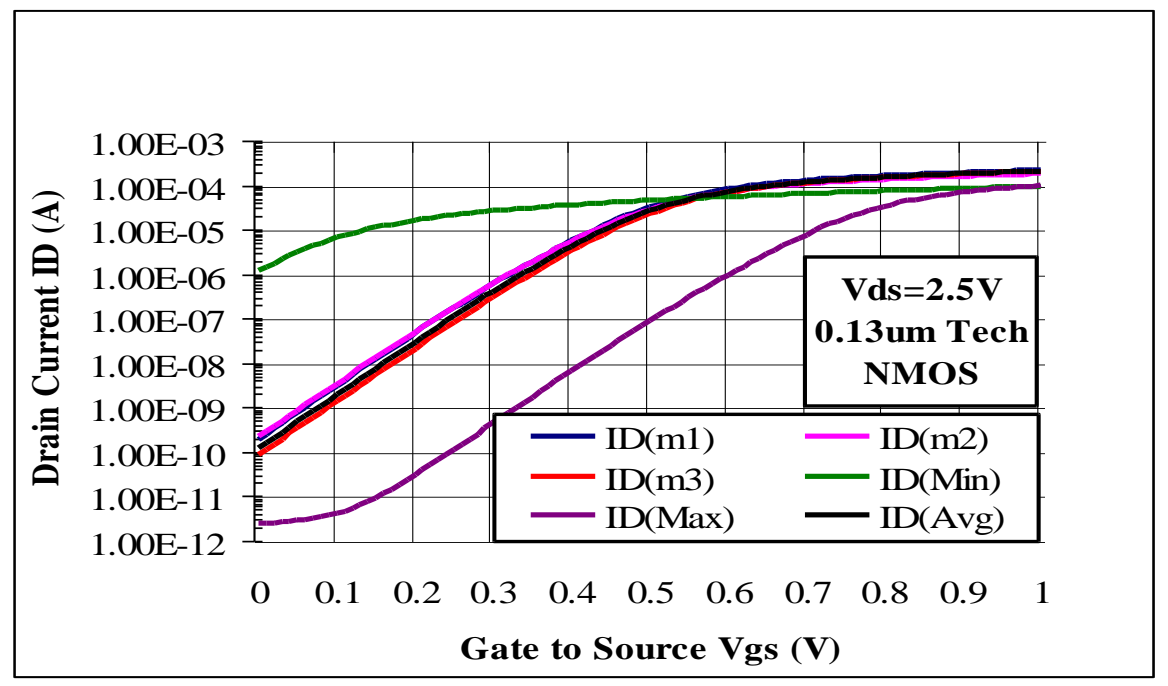

Figure 8: Linear region of NMOS device $(0.5 \mathrm{u} / 0.13 \mathrm{u})$ at high Vds and using model cards for CMOSN1, CMOSN2, CMOSN3 from the portal and the computed CMOSNMIN, CMOSNMAX, and CMOSNA VG model cards using the 140 model cards downloaded from MOSIS portal for NMOS and PMOS 0.13u m Technology.

\subsection{Single De vice Characterization - NMOS}

Fig. 7 and Fig. 8 show the linear Linear region of NMOS device $(0.5 \mathrm{u} / 0.13 \mathrm{u})$ at low and high Vds using model cards for CMOSN1, CMOSN2, CMOSN3 from the portal and the computed CMOSNMIN, CMOSNMAX, and CMOSNAVG model cards using the 140 model cards downloaded from MOSIS portal for NMOS and PMOS 0.13u m Technology.
The NMOS device drain current characteristics is shown on Fig. 7 and The drain and gate conductance are also simulated using the computed models for NMOS device as shown in Fig. 8 and Fig. 9. From Fig. 7-10, it is noted that the CMOSNAVG model card tracks results from CMOSN1, CMOSN2, CMOSN3 results.

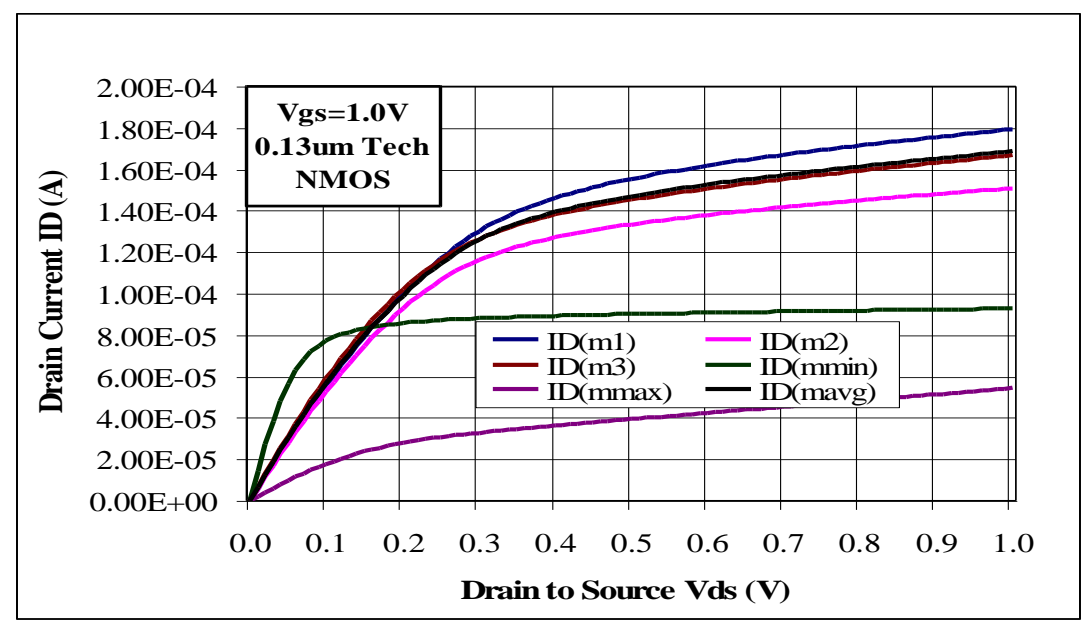

Figure 9: IV characteristics of NMOS device $(0.5 \mathrm{u} / 0.13 \mathrm{u})$ at $\mathrm{Vgs}=1.0 \mathrm{~V}$ and $\mathrm{using}$ model cards for CMOSN1, CMOSN2, CMOSN3 from the portal and the computed CMOSNMIN, CMOSNMAX, and CMOSNA VG model cards using the 140 model cards downloaded from MOSIS portal for NMOS and PMOS 0.13u m Technology. 


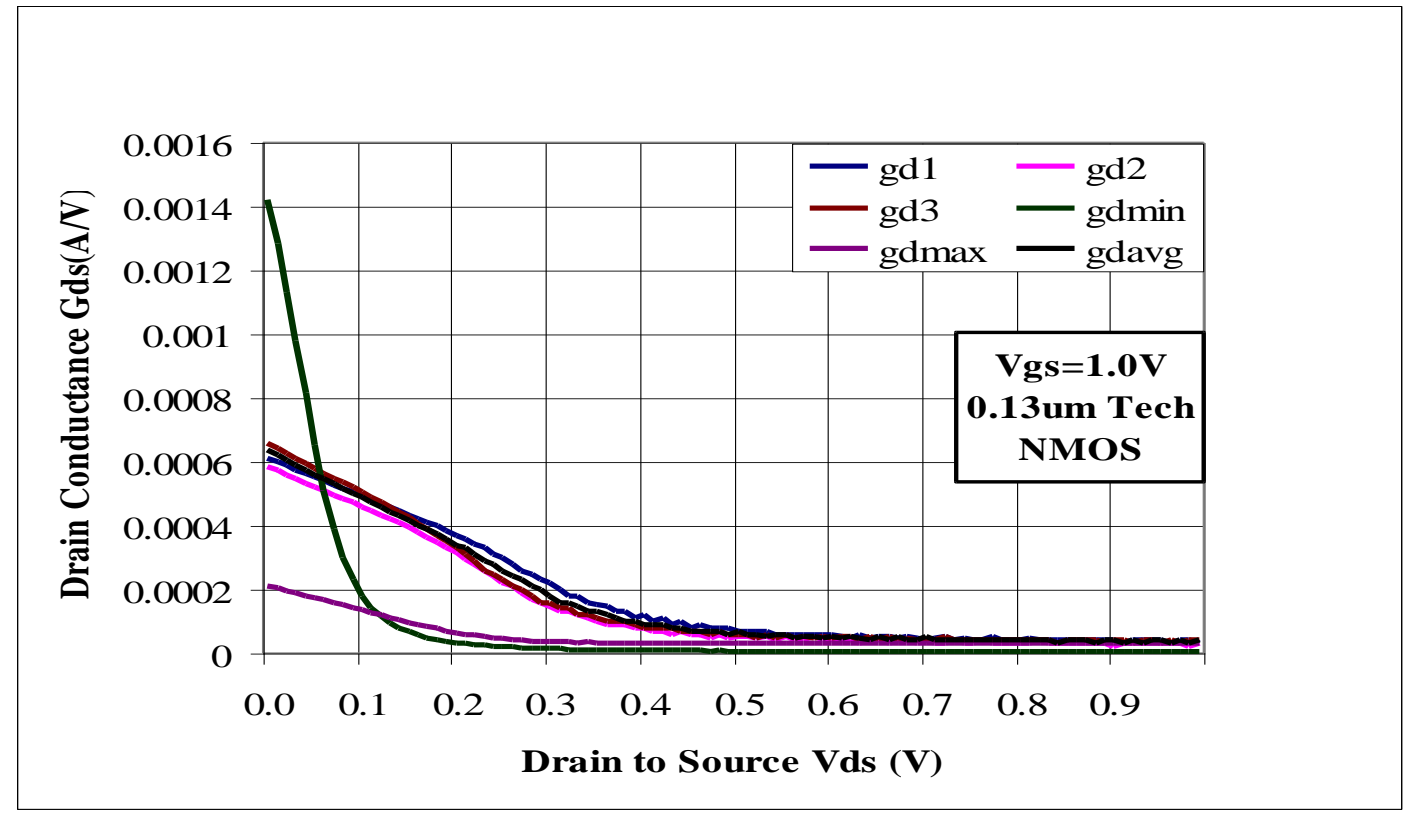

Figure 10: Drain conductance of NMOS device $(0.5 \mathrm{u} / 0.13 \mathrm{u})$ at $\mathrm{Vgs}=1.0 \mathrm{~V}$ and $\mathrm{using}$ model cards for

CMOSN1, CMOSN2, CMOSN3 from the portal and the co mputed CMOSNMIN, CMOSNMAX, and CMOSNA VG model cards using the 140 model cards downloaded from MOSIS portal for NMOS and PMOS 0.13u m Technology.

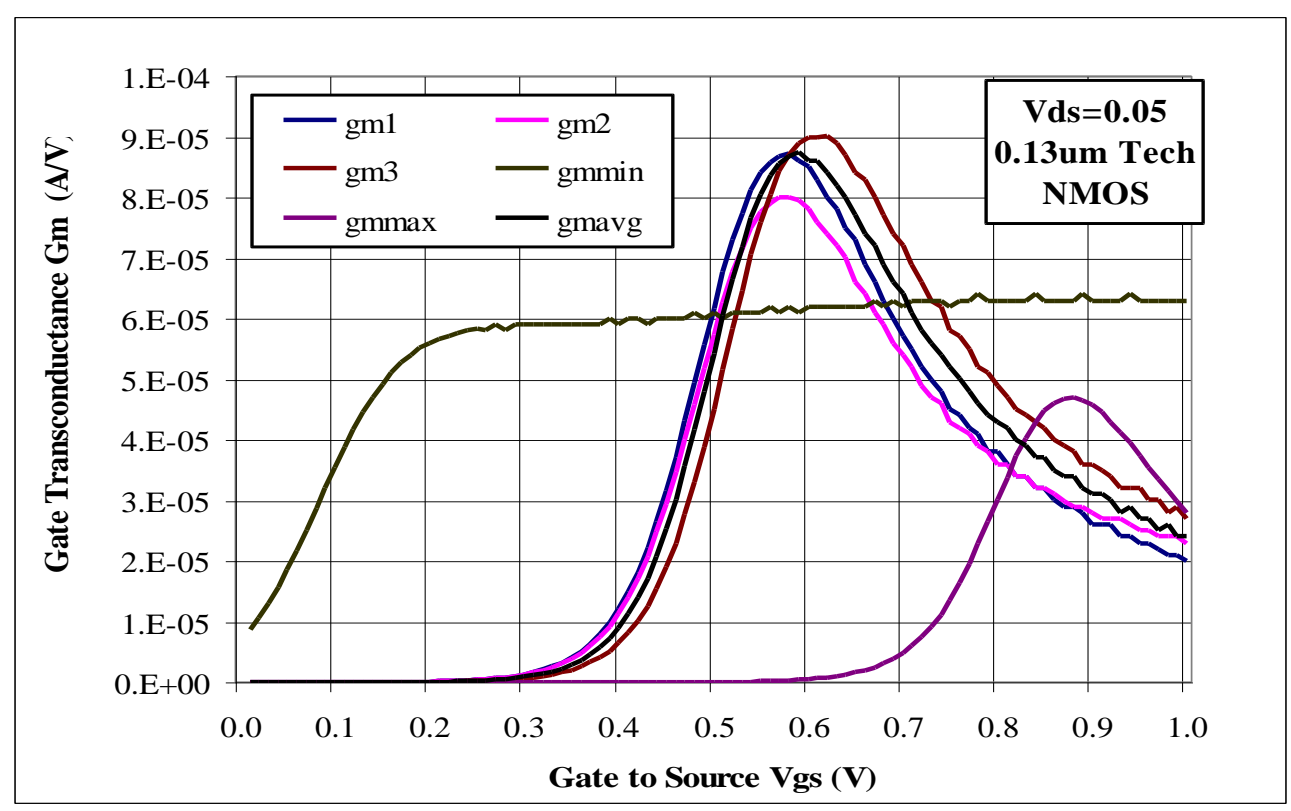

Figure 11: Gate transconductance of NMOS device $(0.5 \mathrm{u} / 0.13 \mathrm{u})$ at $\mathrm{Vds}=0.05 \mathrm{~V}$ and using model cards for CMOSN1, CMOSN2, CMOSN3 from the portal and the computed CMOSNMIN, CMOSNMAX, and CMOSNA VG model cards using the 140 model cards downloaded from MOSIS portal for NMOS and PMOS 0.13u m Technology. 


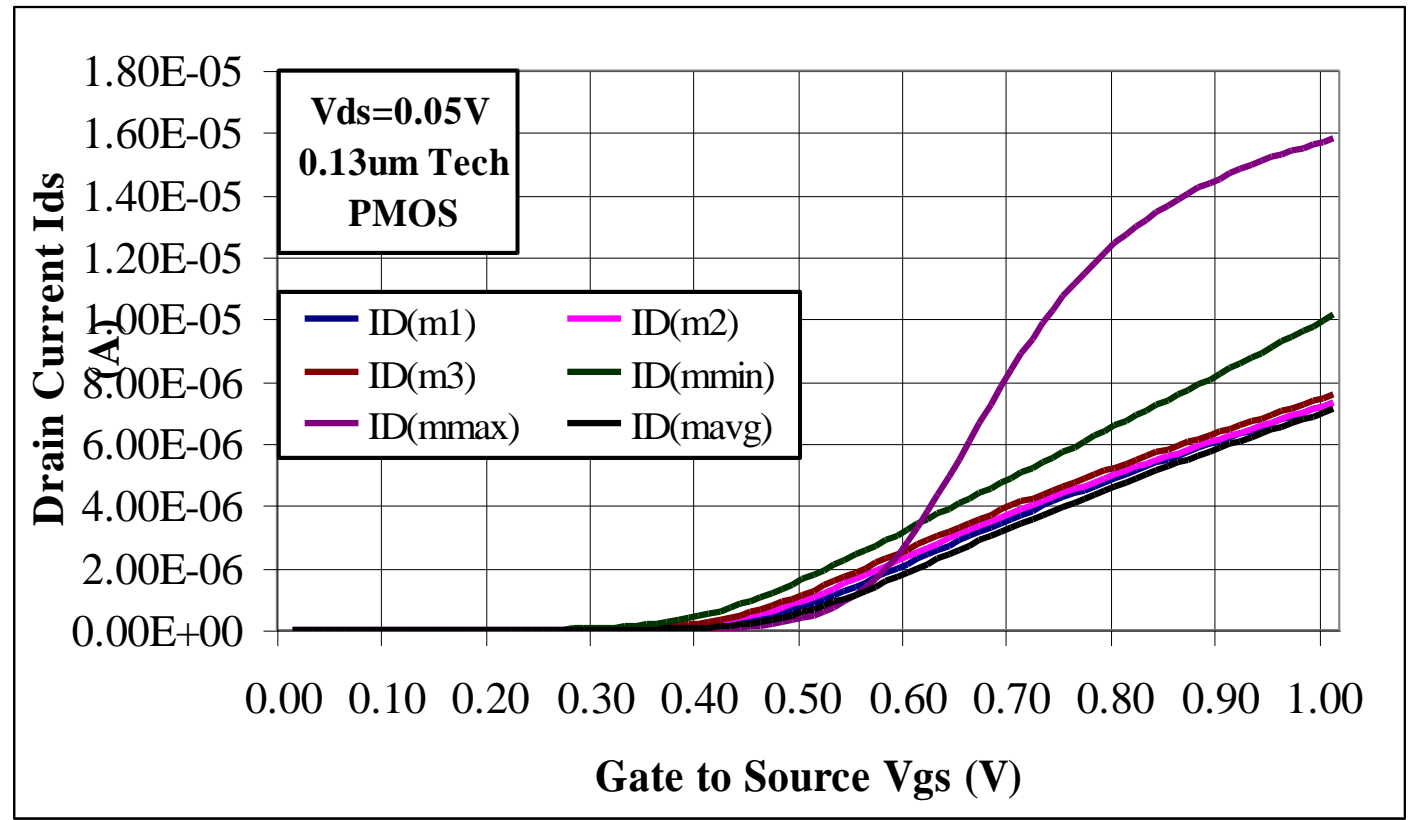

Figure 12: Linear region of PMOS device $(0.5 \mathrm{u} / 0.13 \mathrm{u})$ at low $\mathrm{Vds}=0.05 \mathrm{~V}$ and $\mathrm{using}$ model cards for CMOSP1, CMOSP2, CMOSP3 from the portal and the computed CMOSPMIN, CMOSPMAX, and CMOSPA VG model cards using the 140 model cards downloaded from MOSIS portal for NMOS and PMOS $0.13 \mathrm{um}$ Technology.

The computed CMOSNMIN, CMOSNMAX models results are further away from the average results. This can be attributed to the computed parameters where the minimum shows lowest end of results while the maximum results shows the highest end of simulation results. From these results it can be concluded that if the average model card for simulations it would provide a good feedback on circuit functionality.

\subsection{Single De vice Characterization - PMOS}

A PMOS device of $(0.5 \mathrm{um} / 0.13 \mathrm{um})$ is used to run simulations using model cards for CMOSN1, CMOSN2, CMOSN3 from the portal and the computed CMOSNMIN, CMOSNMAX, and CMOSNAVG model cards using the 140 model cards downloaded from MOSIS portal for NMOS and PMOS 0.13u m Technology.

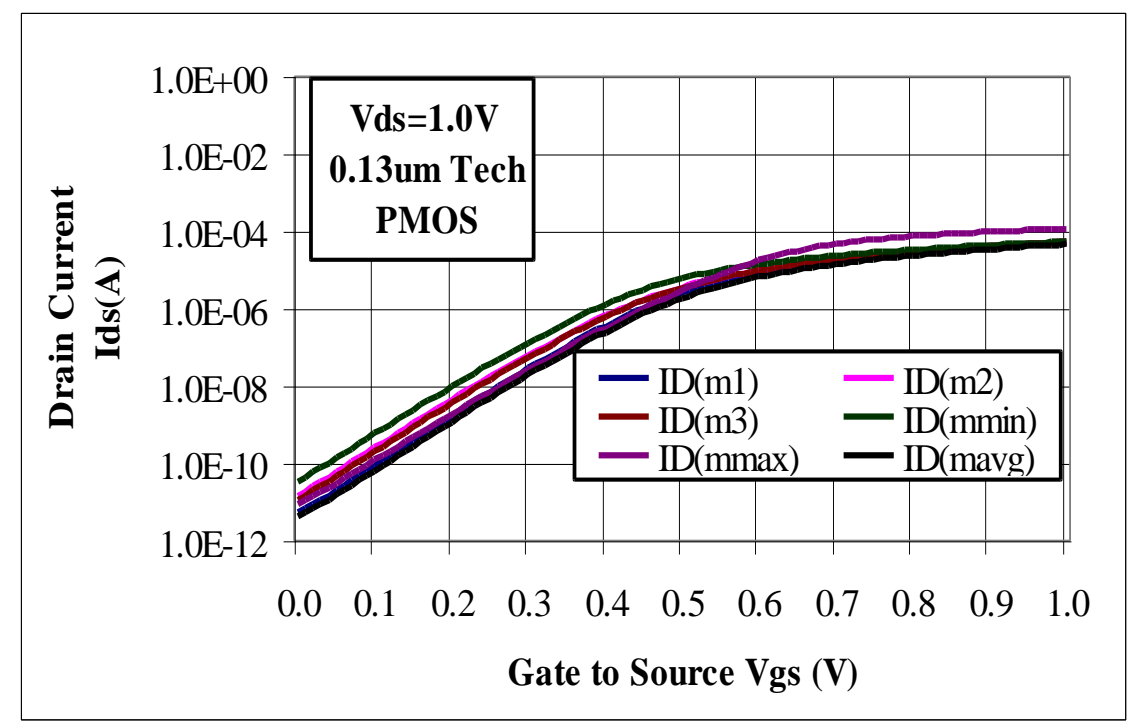

Figure 13: Linear region of PMOS device $(0.5 \mathrm{u} / 0.13 \mathrm{u})$ at high $\mathrm{Vds}=1.0$ Vand using model cards for CMOSP1, CMOSP2, CMOSP3 from the portal and the computed CMOSPMIN, CMOSPMAX, and CMOSPA VG mode1 cards using the 140 model cards downloaded from MOSIS portal for NMOS and PMOS 0.13um Technology. 
The plots shown in Fig. 12 - Fig.16 provide behaviour of PMOS device as predicted by the provided and computed model cards.

The PMOS device characteristics exhibits a similar behaviour observed for the NMOS device. Again, the CMOSPA VG model card tracks results from CMOSP1, CMOSP2, CMOSP3 results. The computed CMOSPMIN, CMOSPMAX models results are tracking low and high end of the model card spectrum and while the average model simulations are showing good representation of the sample model results.

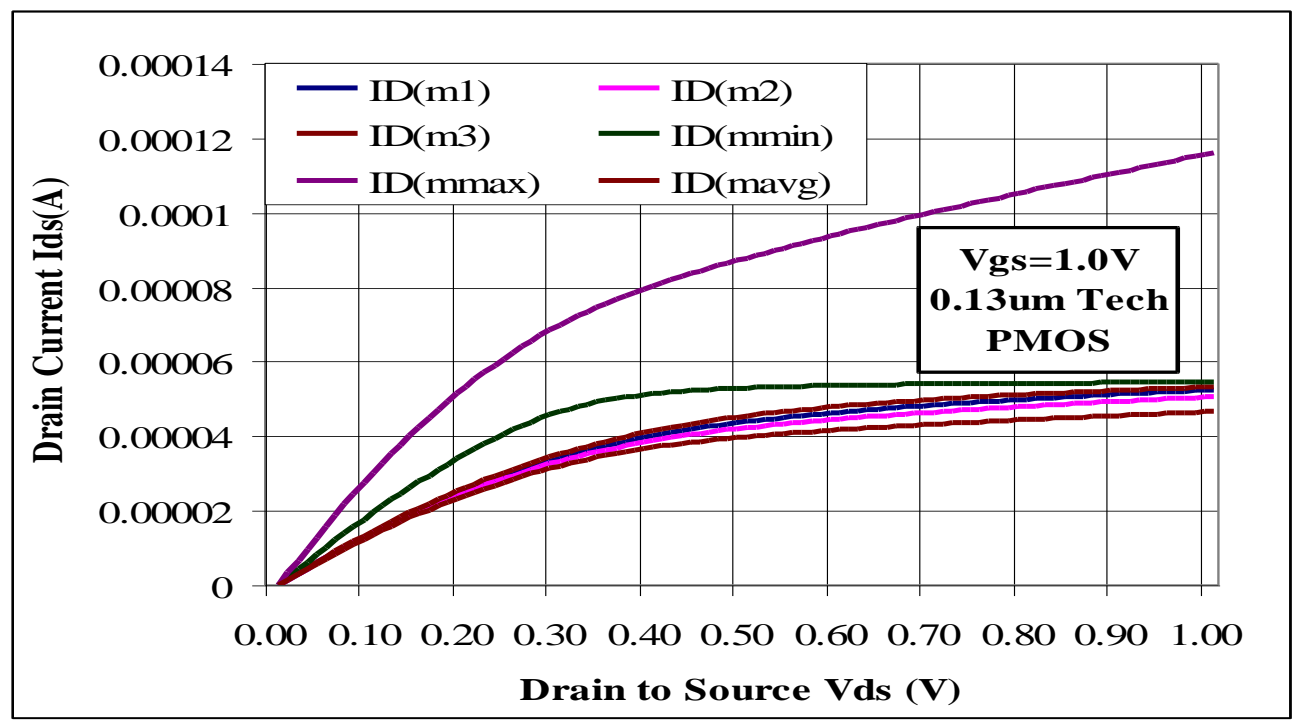

Figure 14: IV characteristics of PMOS device $(0.5 \mathrm{u} / 0.13 \mathrm{u})$ at $\mathrm{Vgs}=1.0 \mathrm{~V}$ and using model cards for CMOSP1, CMOSP2, CMOSP3 fro $m$ the portal and the computed CMOSPMIN, CMOSPMAX, and CMOSPA VG model cards using the 140 model cards downloaded from MOSIS portal for NMOS and PMOS 0.13u m Technology.

Figure 15: Drain conductance of PMOS device $(0.5 \mathrm{u} / 0.13 \mathrm{u})$ at $\mathrm{Vgs}=1.0 \mathrm{~V}$ and $\mathrm{using}$ model cards for CMOSP1, CMOSP2, CMOSP3 from the portal and the computed CMOSPMIN, CMOSPMAX, and CMOSPA VG model cards using the 140 model cards downloaded from MOSIS portal for NMOS and PMOS 0.13u m Technology.

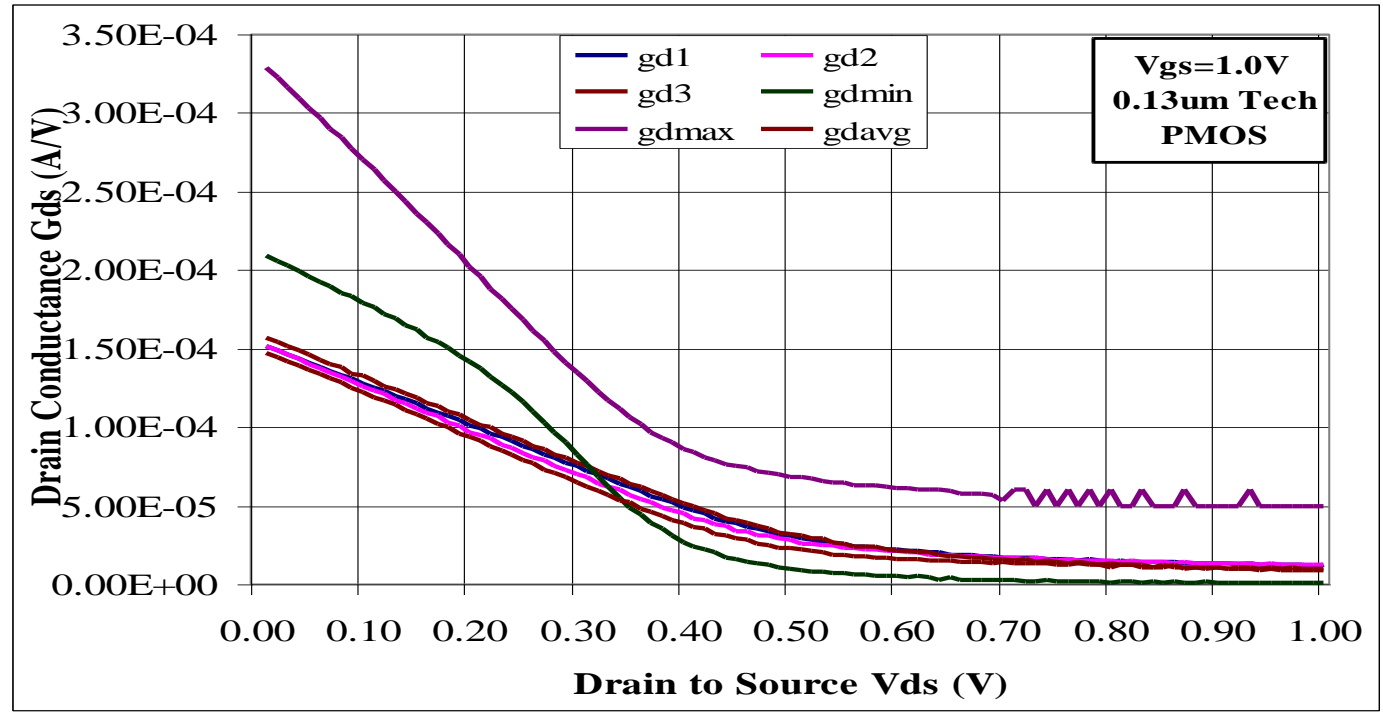




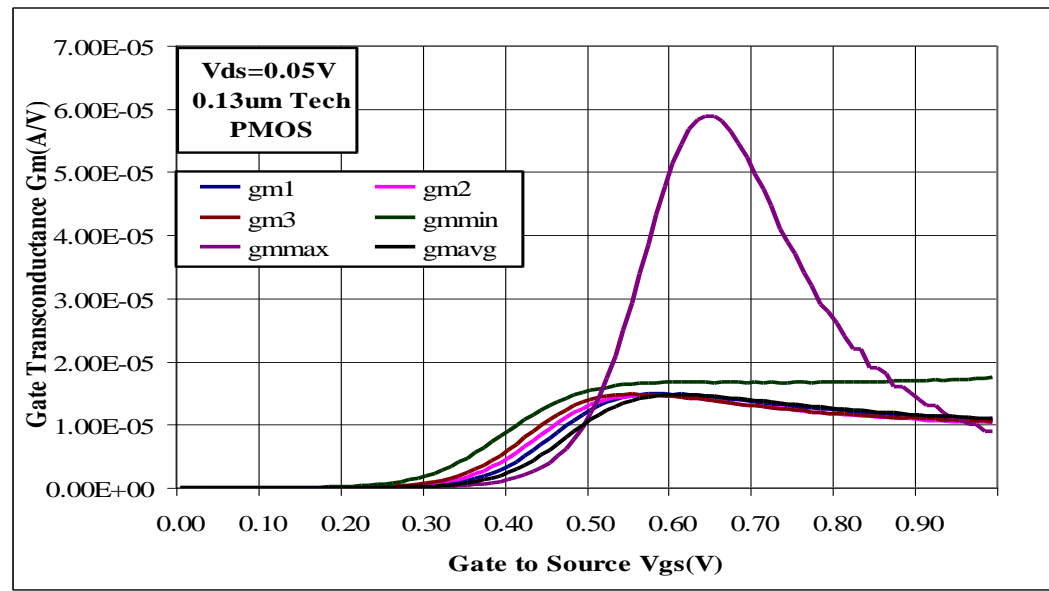

Figure 16: Gate transconductance of PMOS device $(0.5 \mathrm{u} / 0.13 \mathrm{u})$ at $\mathrm{Vds}=0.05 \mathrm{~V}$ and using model cards for CMOSP1, CMOSP2, CMOSP3 from the portal and the computed CMOSPMIN, CMOSPMAX, and CMOSPA VG model cards using the 140 model cards downloaded from MOSIS portal for NMOS and PMOS 0.13u m Technology.

\subsection{Simple Inver ter Simulations}

A simple inverter is constructed using a PMOS device with $(1 \mathrm{u} / 0.13 \mathrm{u})$ and NMOS device with $(0.5 \mathrm{u} / 0.13 \mathrm{u})$ sizes. The netlist of such inverter is shown in Fig. 11. The figure shows the case when the average model name is used for the PMOS and NMOS devices. The netlist is edited for the cases of all of the model names of N1(P1), N2(P2), N3(P3), NMIN(PMIN), NMAX(PMAX) model names. The DC transfer function of the inverter is then plotted while input voltage is swept across rail span from 0 to $2.5 \mathrm{~V}$.

The DC characteristics and the transient response of the inverter are simulated for the models of NMOS and PMOS devices as represented by the netlist file shown in Fig. 17 for PSPICE circuit simulator. The inverter characteristics are plotted as shown in Fig. 18, and Fig. 19 for DC transfer function and transient time characteristics respectively. The average model results are tracking results of models from $0.13 \mathrm{um}$ Technology.

\section{CONCLUSIONS}

Circuit simulations of single devices and simple circuits are performed using spice models downloaded from MOSIS fabrication services portal site. Several technology models were experimented with. To reduce the number of simulations required, it is found that user can produce an average model card by averaging the model parameters of all device model avaliable. Similarly, min mum and maximum model cards can e produced using the MIN and MAX functions of tabular data. In this work, a total of 140 models for NMOS and PMOS devices from $0.13 \mathrm{um}$ Technology were available for simulations. The model cards were used to simulate NMOS and PMOS device characteristics in the linear region, and saturation regions. Additionally, the single device gate transconductance, and drain conductance are also tested. It was found that the average model card produces results that closely represent sample model cards. To further test validity of using average model, an inverter circuit is simulated for DC

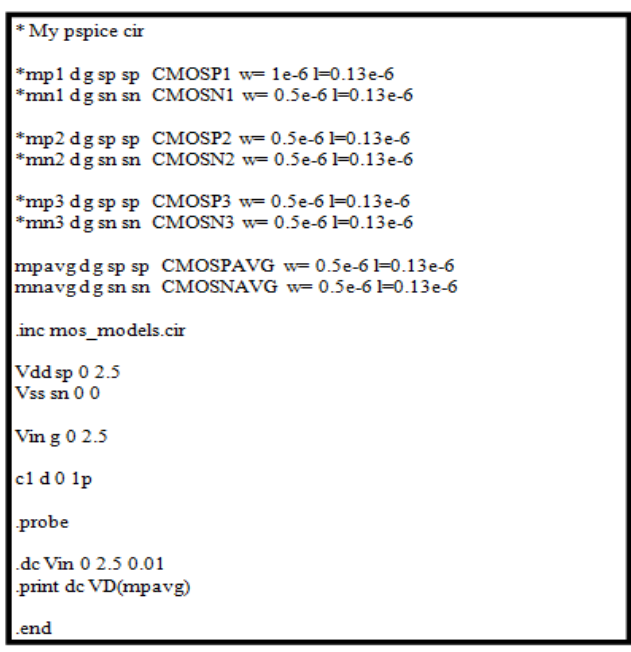

Figure 17: PSPICE net list file of a simple inverter using NMOS and PMOS devices from $0.13 \mathrm{um}$

Technology to evaluate provided and computed model cards behaviour. 


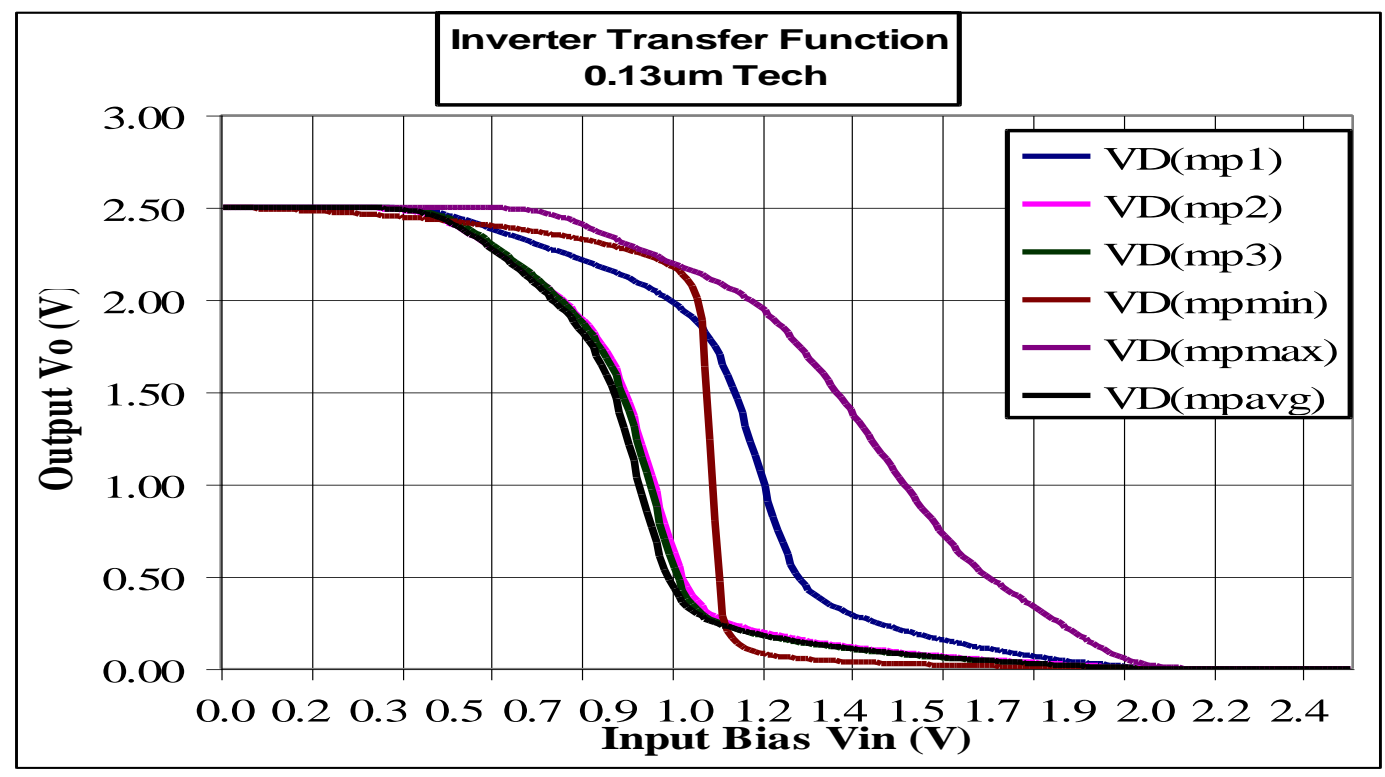

Figure 18: Inverter DC transfer characteristics simulated using the provided and computed models. transfer function and transient response. The average model response exhibit close fit to behaviour from sample model cards.

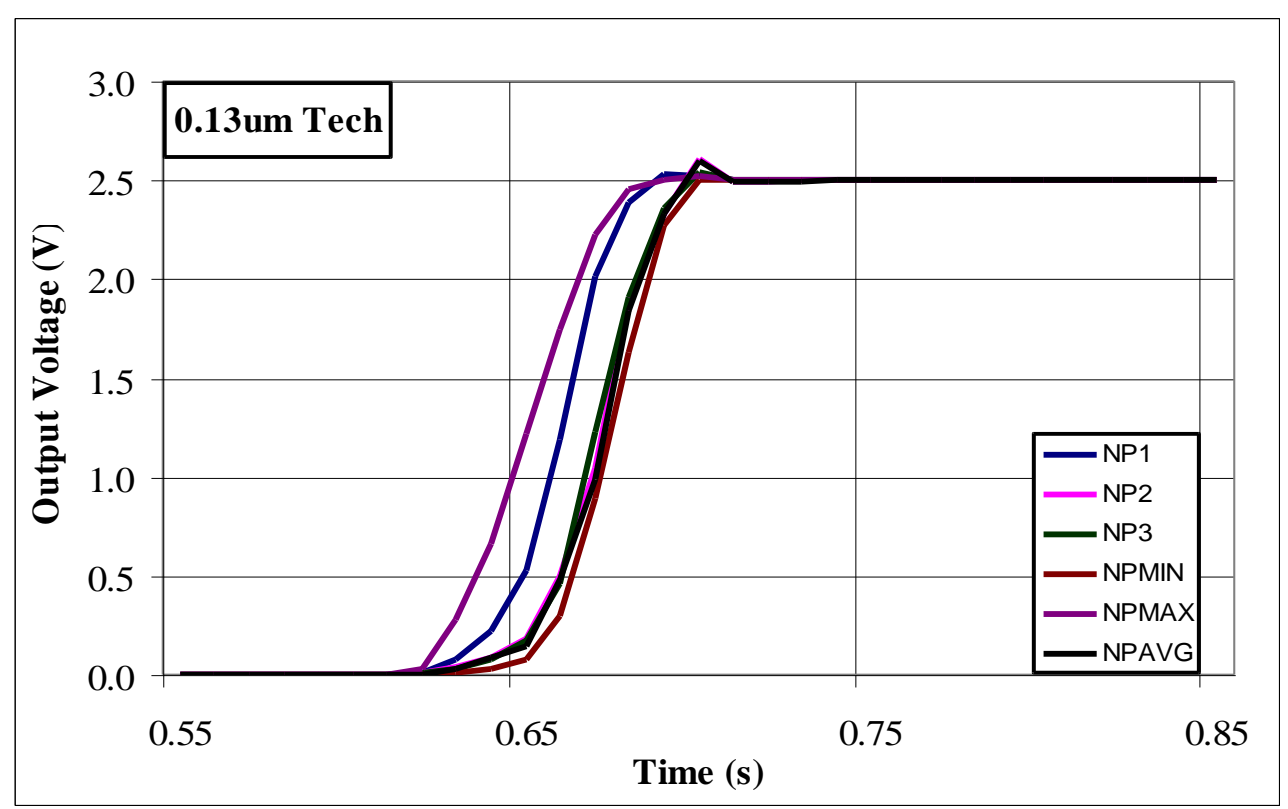

Figure 19: Inverter transient characteristics simulated using the provided and computed models.

\section{REFERENCES}

[1]. MOSIS Integration Circuit Fabrication Service, "https://www.mosis.com".

[2]. Ashraf Os man, A min B. Abdel Nabi, Is mail El-A zhary, "The Potential of Establishing Computer_Aided_Design (CAD) Industry in Africa-Sudan As a Case-Study”, IEEE, 2013 International Confe rence on Computing, Electrical and Electronics Engineering (ICCEEE), 26-28 Aug. 2013, pp 87-91. 